EUROPEAN ORGANIZATION FOR NUCLEAR RESEARCH

CERN - AB DEPARTMENT

CERN-AB-2006-003

\title{
OBSERVATION OF OCTUPOLE DRIVEN RESONANCE PHENOMENA WITH SPACE CHARGE AT THE CERN PROTON SYNCHROTRON
}

\author{
E. Métrala, G. Franchettib, M. Giovannozzia ${ }^{\text {, I. Hofmann }}{ }^{\text {b }}$, M. Martinia and R. Steerenberga
}

\begin{abstract}
Several benchmarking space charge experiments have been performed during the last few years in the CERN Proton Synchrotron. These controlled experiments are of paramount importance to validate the present very powerful simulation codes. The observations of the combined effect of space charge and nonlinear resonance on beam loss and emittance, using a single controllable octupole during $\sim 1 \mathrm{~s}$ at $1.4 \mathrm{GeV}$ kinetic energy, are discussed in some detail in the present paper. By lowering the working point towards the octupolar resonance, a gradual transition from a regime of loss-free core emittance blow-up to a regime of continuous loss was found.
\end{abstract}

Presented at COULOMB'05 workshop on High Intensity Beam Dynamics, Senigallia (AN), Italy, September 12-16, 2005

a CERN, Geneva, Switzerland

b GSI, Darmstadt, Germany

Geneva, Switzerland

10 January 2006 


\title{
Observation of Octupole Driven Resonance Phenomena with Space Charge at the CERN Proton Synchrotron
}

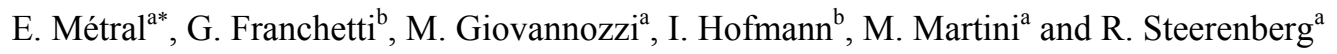 \\ ${ }^{a}$ CERN, 1211 Geneva 23, Switzerland \\ ${ }^{\mathrm{b}}$ GSI, 64291 Darmstadt, Germany
}

Several benchmarking space charge experiments have been performed during the last few years in the CERN Proton Synchrotron. These controlled experiments are of paramount importance to validate the present very powerful simulation codes. The observations of the combined effect of space charge and nonlinear resonance on beam loss and emittance, using a single controllable octupole during $\sim 1 \mathrm{~s}$ at $1.4 \mathrm{GeV}$ kinetic energy, are discussed in some detail in the present paper. By lowering the working point towards the octupolar resonance, a gradual transition from a regime of loss-free core emittance blow-up to a regime of continuous loss was found.

\section{Introduction}

Space charge tune shift can drive the beam onto linear and/or non-linear resonances generating transverse emittance blow-up and sometimes subsequent beam loss. Depending on how the space charge tune spread overlaps the resonance, i.e. whether the centre or the tail of the particles distribution is in the stop-band, the beam behaviour will be completely different. The first case leads to a loss-free (if the mechanical aperture is sufficiently large) core-emittance blow-up, where the distribution is almost conserved [1]. The second leads to a diffusion of the tail particles and a subsequent beam loss [2-4]. The explanation of the beam loss regime is in terms of the synchrotron oscillation, which causes a periodic tune modulation due to space charge, and leads to trapping and detrapping on the resonance islands [2]. For working points very close to the resonance this induces a beam halo with large radius, which in conjunction with the reduced dynamic aperture is the source of beam losses.

The two cases are sketched in Fig. 1, where the space charge tune spread has been computed using Keil's formula, which considers a bi-Gaussian in the horizontal and vertical plane [5]. To obtain a reasonable accuracy up to $2.5 \sigma$ of the distribution the field description has been pushed up to the $15^{\text {th }}$ order. A gap without particles between the low-intensity working point and the incoherent working points is observed as the third (longitudinal) distribution is not taken into account (see Ref. [6] where a tri-Gaussian in the horizontal, vertical and longitudinal plane is considered).

\footnotetext{
${ }^{*}$ Corresponding author: Elias.Metral@cern.ch.
}
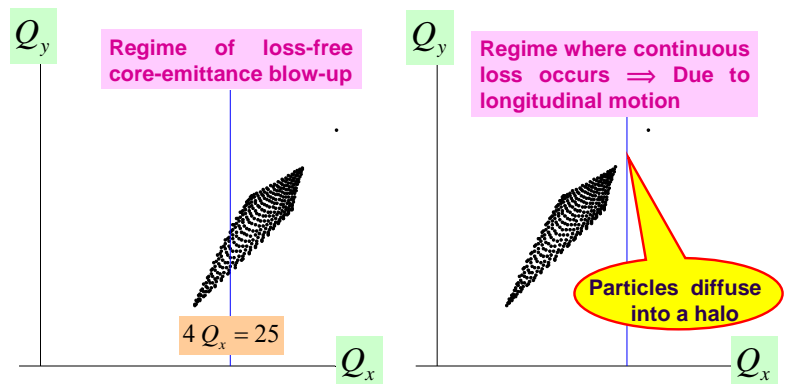

Figure 1. Core emittance growth dominated (left) and continuous beam loss dominated (right) regimes.

The first evidence of single-particle motion near islands has been observed at the Indiana University Cyclotron Facility cooler ring [7]. The effect of machine tune modulation on island trapping or detrapping has also been studied experimentally at the CERN Super Proton Synchrotron (SPS) [8,9], where the modulation was found to lead to diffusion effects, and at the Fermilab Tevatron [10], but all these studies were performed in the absence of space charge. The main point of the present work is to study these phenomena in the context of the intrinsic tune modulation caused by space charge and synchrotron motion. Additional complexity is therefore introduced due to the following features: (i) the tune of an individual particle is strongly modulated, by an amount comparable with the incoherent tune shift, depending on two parameters, the synchrotron phase as well as the betatron amplitude, (ii) the latter is itself evolving in time, depending on the preceding trapping and detrapping events, and (iii) there is a self-consistent time evolution due to the global 
changes of space charge. Experimental verifications of single-particle behaviour are not possible under these circumstances. Instead a 3D computer simulation with space charge is used to demonstrate the long-term balance of trapping and detrapping as competing mechanisms and to compare the global predictions on the rms emittance and halo growth with the measurements.

After a brief review of the CERN Proton Synchrotron (PS) lattice and main magnet in Section 2, the experimental results of two measurement campaigns made in 2002 and 2003 are presented in Sections 3 and 4 respectively. A detailed analysis of the first measurements, focusing essentially on the emittance growth dominated regime, has already been published in Ref. [2]. The second, more refined, measurement campaign is reported here for the first time, giving more emphasis on the beam loss dominated regime. The experimental setup is presented in Fig. 2, and the relevant machine and beam parameters are summarized in Table 1. The space charge was chosen significantly

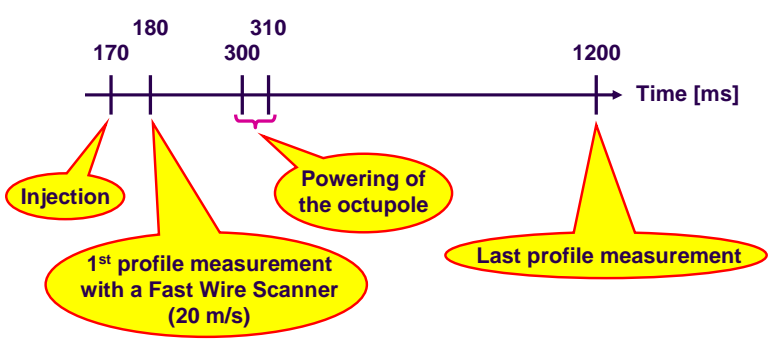

Figure 2. Experimental setup.

Table 1

Basic beam and PS parameters relevant for the experiments.

\begin{tabular}{|c|c|c|}
\hline Parameter & Value & Unit \\
\hline Circumference & 628 & $\mathrm{~m}$ \\
\hline $\begin{array}{l}\text { Horiz. / vert. beam pipe } \\
\text { diameter } \\
\end{array}$ & $14 / 7$ & $\mathrm{~cm}$ \\
\hline Superperiodicity & 10 & \\
\hline Kinetic energy & 1.4 & $\mathrm{GeV}$ \\
\hline Vert. tune & 6.12 & \\
\hline Horiz. tune & $6.24<Q_{x}<6.32$ & \\
\hline $\begin{array}{c}\text { Horiz. / vert. relative } \\
\text { chromaticities }\end{array}$ & $\sim-1 / \sim-1$ & \\
\hline \# of bunches & 1 & \\
\hline$\#$ of protons per bunch & $\sim 10^{12}$ & \\
\hline Bunch length $(4 \sigma)$ & $\sim 180$ & ns \\
\hline $\begin{array}{l}\text { Horiz. / vert. normalised } \\
\text { rms emittances }\end{array}$ & $\sim 5 / \sim 2.5$ & $\mathrm{~mm} \mathrm{mrad}$ \\
\hline Rms momentum spread & $1.4 \times 10^{-3}$ & \\
\hline $\begin{array}{c}\text { Horiz. / vert. rms beam } \\
\text { sizes }\end{array}$ & $\sim 7 / \sim 4$ & $\mathrm{~mm}$ \\
\hline Derived small- & $\sim-0.075 / \sim-0.125$ & \\
\hline
\end{tabular}

\begin{tabular}{|c|c|c|}
\hline $\begin{array}{c}\text { amplitudes horiz. /vert. } \\
\text { space charge tune shifts }\end{array}$ & \\
\hline Synchrotron period & $\sim 1.5$ & $\mathrm{~ms}$ \\
\hline $\begin{array}{c}\text { Octupole strength }\left(K_{3}\right) \\
\text { with } I_{\text {oct }} \text { the current }\end{array}$ & $1.215 I_{\text {oct }}$ & $\mathrm{m}^{-3}$ \\
\hline $\begin{array}{c}\text { Horiz. / vert. betatron } \\
\text { functions at the octupole }\end{array}$ & $11.7 / 22.2$ & $\mathrm{~m}$ \\
\hline $\begin{array}{c}\text { Betatron function for } \\
\text { horiz. emittance meas. }\end{array}$ & 12.6 & $\mathrm{~m}$ \\
\hline $\begin{array}{c}\text { Dispersion function for } \\
\text { horiz. emittance meas. }\end{array}$ & 2.3 & $\mathrm{~m}$ \\
\hline $\begin{array}{c}\text { Betatron function for } \\
\text { vert. emittance meas. }\end{array}$ & 11.6 & $\mathrm{~m}$ \\
\hline
\end{tabular}

below the maximum possible value in the CERN PS to avoid overlap with other resonances. The vertical machine tune was set to $Q_{y}=6.12$, and the horizontal one was varied in the interval $6.24<Q_{x}<6.32$. The relative transverse chromaticities were close to the natural ones $(\sim-1)$. The kinetic energy was kept at the injection value of $1.4 \mathrm{GeV}$ (corresponding to a beam momentum of $2.14 \mathrm{GeV} / \mathrm{c}$ ) with a measurement window of $\sim 1$ s (i.e. $\sim 4.4 \times 10^{5}$ turns) over which the bunch intensity was monitored with a current transformer. The calibrated octupole (here $K_{3}=1.215 I_{o c t} \mathrm{~m}^{-3}$, which is the integrated octupole strength over the octupole magnetic length divided by the beam rigidity) was powered after injection to excite the resonance $4 Q_{x}=25$. In fact this octupole is composed of 2 octupoles in series powered by a single power supply placed in section 20 of the PS with $K_{3}=0.6075 I_{o c t} \mathrm{~m}^{-3}$ each. The transverse profiles were measured with flying wires (at $20 \mathrm{~m} / \mathrm{s}$ ), fitted with a Gaussian profile to determine the corresponding rms emittances. Initial and, in most cases, final profiles were actually found to be quite close to Gaussian distributions (in horizontal, which is the plane of interest), as will be seen below. The two transverse planes were decoupled by setting a current in the skew quadrupoles of $0.33 \mathrm{~A}$ [11].

\section{PS lattice and main magnet}

The PS lattice consists of ten super-periods each made of ten combined-function magnets $4.4 \mathrm{~m}$ long, interlaced with eight $1.6 \mathrm{~m}$ and two $3.0 \mathrm{~m}$ drift spaces. The control of the linear tunes as well as the chromaticities in both transverse planes, i.e. four parameters, is achieved by means of the three currents of special coils added to the main magnets, namely two pole-face-windings and one figure-of-eight loop. The overall magnetic field configuration is therefore rather complicated and a linear model of the PS main magnet does not provide sufficient precision to model correctly the particle dynamics. Accurate measurements of the nonlinear tune as a function of both amplitude and momentum offset have been performed to extract both 
linear and nonlinear properties of the lattice [12], following a method previously applied in the SPS [13]. The measurement technique is the following. Both horizontal and vertical tunes are measured as functions of the momentum offset generated by a proper RFperturbation. A polynomial fit of the measured curve is then performed to extract numerical information on the different orders. Virtual nonlinear elements (located at both dipole ends), represented by thin lens elements, are inserted in the machine lattice model and they are used as fit parameters to reproduce the fitted polynomial. This procedure is applied order-by-order, i.e. the quadrupolar components are used to reproduce the constant term in the polynomial, the sextupolar components the linear term and so on, up to the dodecapolar components.

The summary of the values of the fitted multipolar components is presented in Table 2 for the injection beam momentum, the expansion of the magnetic field in multipoles being

$$
B_{y}+i B_{x}=B_{0} \rho_{0} \sum_{i=1}^{M}\left[K_{n}+i J_{n}\right] \frac{(x+i y)^{n}}{n !}-B_{0}
$$

with

$$
K_{n}=\frac{l}{B_{0} \rho_{0}} \frac{\partial^{n} B_{y}}{\partial x^{n}}, \quad J_{n}=\frac{l}{B_{0} \rho_{0}} \frac{\partial^{n} B_{x}}{\partial x^{n}}
$$

where $\rho_{0}$ is the nominal bending radius and $l$ the length of the magnetic element. The values quoted in Table 2 are for one magnet. The one hundred PS magnets thus lead to a $D$-component of $K_{3}=-17 \mathrm{~m}^{-3}$ which is distributed around the machine. In comparison, as will be seen in the two measurement campaigns, currents in the single localized controllable octupole of $+40 \mathrm{~A}$ and $-20 \mathrm{~A}$ were used, leading to $K_{3}=48.6 \mathrm{~m}^{-3}$ and $K_{3}=-24.3 \mathrm{~m}^{-3}$ respectively.

Table 2

Summary of the virtual kick values ( $F$ and $D$ stand for the focusing and defocusing half-unit types of the PS magnet) for the injection beam momentum.

\begin{tabular}{|c|c|c|c|c|}
\hline $2.14 \mathrm{GeV} / \mathrm{c}$ & $K_{1}\left(10^{-4}\right)$ & $K_{2}$ & $K_{3}$ & $K_{4}$ \\
\hline$F$ & -10.3 & 0.013 & 0.026 & -105.53 \\
\hline$D$ & 10.2 & -0.0014 & -0.17 & 150.79 \\
\hline
\end{tabular}

\section{First measurements (14-18 October 2002)}

The measurements were carried out as part of a highintensity machine development time at the PS in October 2002. In Fig. 3, the results of final measurements at $1.2 \mathrm{~s}$ are plotted as a function of the machine horizontal tune.

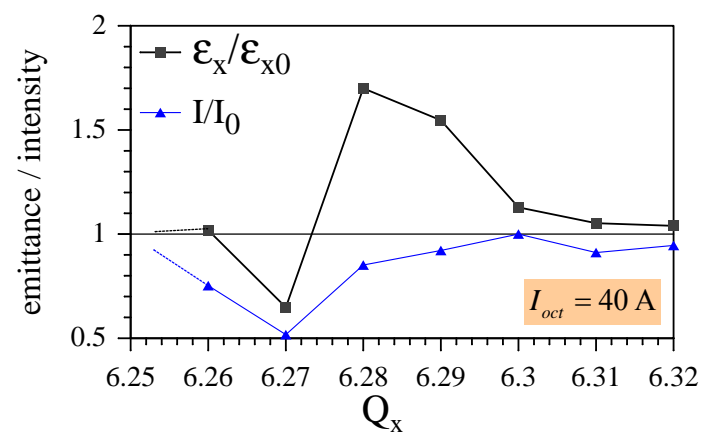

Figure 3. Experimental results of the final rms emittances (of Gaussian fit) and beam current relative to initial values.

The existence of two regimes is clearly revealed in Fig. 3. An emittance growth dominated regime for $Q_{x}$ sufficiently above the resonance (in our example $Q_{x}>$ 6.28 ) and a loss dominated regime for $Q_{x}<6.28$. It is noted that for the working point of maximum beam loss $\left(Q_{x}=6.27\right)$ the emittance also shrinks, since large amplitude particles are predominantly lost. The time evolution of the bunch intensity for $Q_{x}=6.27$ is shown in Fig. 4. Note the continuous loss at a nearly constant rate after an initially enhanced loss (the intensity drop at $1.2 \mathrm{~s}$ is caused by a kicker event). About $50 \%$ of beam losses were measured $\sim 1 \mathrm{~s}$ after the powering of the octupole.

While the emittance growth dominated regime was in good agreement with predictions, the losses were much larger than expected [2]. It was then decided to make more refined measurements in 2003, to further deepen the understanding of the underlying complex nonlinear dynamics processes, and use the "real" nonlinear model of the PS machine for the simulations instead of the constant focusing approximation used to analyze the 2002 measurements.

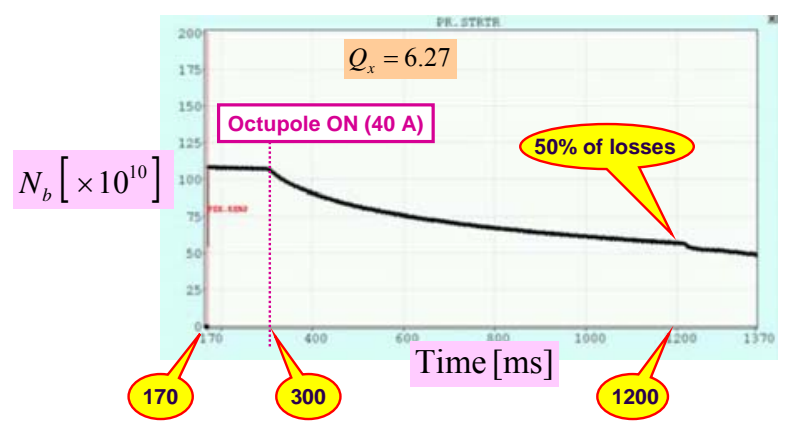

Figure 4. Measured bunch intensity as a function of time for $Q_{x}=6.27$. The initial number of protons in the bunch before the powering of the octupole is $\sim 10^{12}$. The intensity drop at $1200 \mathrm{~ms}$ is caused by a kicker event. 


\section{Second measurements (22-29 September 2003)}

In this second experiment campaign, we first started to make a scan on the octupole current (see Fig. 5) and then decided to choose an octupole current of $-20 \mathrm{~A}$, instead of +40 A used previously. The change of sign of the octupole current was motivated to have a positive detuning with betatron amplitude, which thus adds to the space charge one. The smaller absolute value of the octople current was used to keep a sufficiently large dynamic aperture. Note that the detuning of the octupole and space charge acts in the same direction (they reduce the nominal tune) but in a different way: the space charge detuning is stronger on axis whereas the octupole detuning is stronger off axis. The same kind of plot as in Fig. 3 was then recovered, as shown in Fig. 6, where it is found that the emittance growth regime is in the interval $6.28<Q_{x}<6.32$, with the maximum emittance growth of $42 \%$ at the tune $Q_{x}=6.28$. The beam loss regime is located in the interval $6.25<Q_{x}<6.28$, with the maximum beam loss of $32 \%$ at the tune $Q_{x}=6.265$.

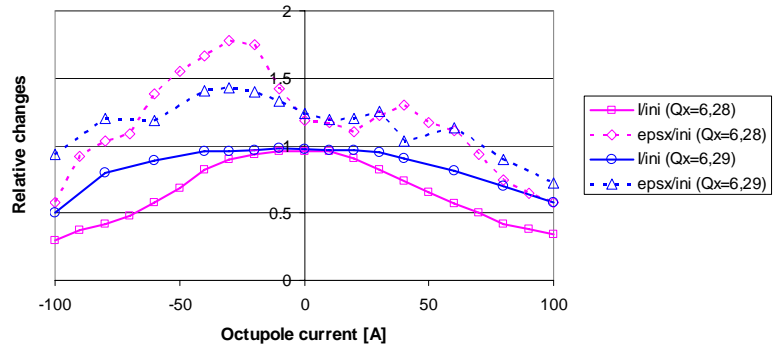

Figure 5. Scan of intensity and horizontal emittance variations (relative to initial values) as a function of the octupole current. Here only, the vertical emittance was in fact equal to the horizontal one. The measurements were made at $1200 \mathrm{~ms}$.

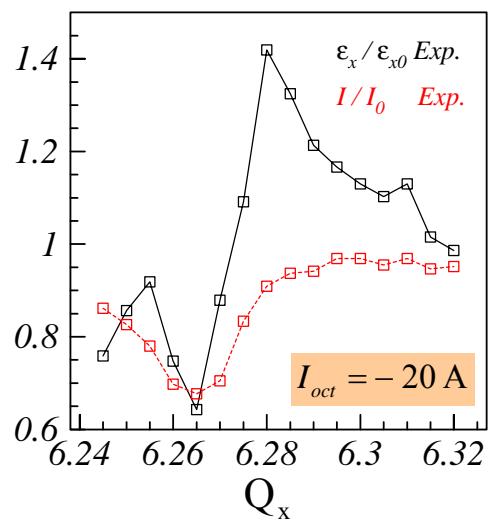

Figure 6. Experimental findings: normalised final emittance and beam intensity as function of the working point (similar plot as in Fig. 3).
More refined measurements than in 2002 have been performed and are reported from Figs. 7 to 25 . The time evolution of the transverse emittances (deduced from Gaussian fits of the transverse profiles) is shown in Fig. 7 in the emittance growth dominated regime, and in Fig. 12 in the beam loss dominated regime. In Figs. 8 and 13 the evolution of the horizontal rms beam size, deduced from a Gaussian fit or from direct rms computation, is shown, revealing that the Gaussian fit is indeed a good approximation, as can also be seen in Figs. 10, 11, 15 and 16. The time evolution of the horizontal profiles is shown in Fig. 9 for the emittance growth dominated regime, and in Fig. 14 for the beam loss dominated regime, where the shrinkage of the horizontal beam size is clearly seen: particles with large positive amplitudes are removed. The time evolution of the longitudinal profiles in the beam loss dominated regime is shown in Fig. 17, with Gaussian fits in Fig. 18 and 19 , which suggests that the lost particles make the longitudinal distribution shrink in amplitude and size. In order to quantify this visual pattern we computed the time evolution of the bunch length by performing a Gaussian fit of the longitudinal bunch profiles of Fig. 17. These results are plotted in Fig. 20. In the same picture the integrated intensity of the longitudinal bunch profile is also plotted. The two curves of Fig. 20 show that there is a direct relation between beam loss and bunch shortening. Unless an unexpected transverselongitudinal correlation takes place, Fig. 20 suggests that the lost particles are not only those with large transverse amplitude, but also with large synchrotron amplitude. This experimental evidence is consistent with the condition of periodic resonance crossing. In fact particles with small longitudinal and transverse amplitudes will always have an effective tune $Q_{x}$ sufficiently below 6.25 , because their motion is confined in the denser region of the bunch, hence they will never be extracted. Particles with large longitudinal amplitude, instead, can periodically cross the resonance and therefore may get trapped and eventually get lost. Note that the curves chosen to fit the measured data are similar to the ones used to fit the simulation results $[3,4]$.

Normalised final (i.e. measured at $1200 \mathrm{~ms}$ ) emittance and beam intensity as function of the working point for the cases (i) with octupole $\mathrm{ON}$ and a current in the octupole of $-20 \mathrm{~A}$, (ii) without (w/o) octupole, and (iii) without (w/o) octupole and with a debunched (deb) beam (i.e. with low space charge) are shown in Fig. 21. The short term Dynamic Aperture (DA) $\left(10^{3}\right.$ turns) is $9 \sigma$ for $I_{\mathrm{oct}}=0 \mathrm{~A}$. By activating the octupole with $I_{\mathrm{oct}}=$ $20 \mathrm{~A}$, the short term DA is lowered to $8 \sigma$ for $6.27<Q_{x}$ $<6.32$, but near $Q_{x}=6.25$ it shrinks to $3.5 \sigma$. This value is small enough to intercept the tail of a Gaussian distribution. A further inspection at $10^{5}$ turns shows that 
close to $Q_{x}=6.25$ the DA without space charge shrinks from $3.5 \sigma$ to $3 \sigma$. Again these results suggest that the DA alone as modelled here and ignoring space charge cannot explain the observed beam losses of $\sim 30 \%$ (see Fig. 21). We also note the significant difference between the study of the single particle stability, where the minimum DA is located at $Q_{x}=6.25$, from the experimental finding of Fig. 6, where the maximum loss occurs at $Q_{x}=6.265$.

We have measured the loss at $Q_{x}=6.265$ after $4.4 \times 10^{5}$ turns with the octupole OFF and found $20 \%$, compared with the $\sim 30 \%$ for the octupole ON (see Fig. 21). The no-octupole measurement showed, however, no emittance growth regime, which suggests that the natural lattice octupoles are too weak to cause efficient trapping/detrapping. Hence, the loss difference between octupole ON/OFF, about $10 \%$, should mainly be due to trapping/detrapping by the additional octupole. This loss difference is in much better agreement with the simulation result of maximum $8 \%$ loss with the octupole ON $[3,4]$. The loss observed in the experiment must be related to the shrinking of the dynamic aperture, since the beam was too small to hit a physical aperture. Note that for a debunched beam (i.e. with low space charge) with no octupole, some losses are already measured together with an increase of the vertical emittance, which may explain why more losses than predicted from simulations are observed (see Figs. 21-25). This effect should be studied in more detail as during the first measurement campaign in 2002 with $+40 \mathrm{~A}$ in the controlled octupole, neither beam losses nor emittance growth were observed.

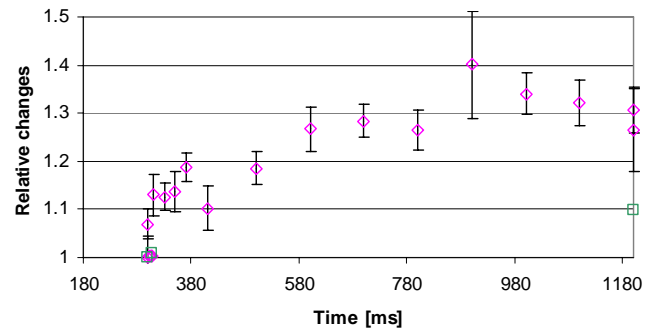

$\diamond$ epsx/ini $\square$ epsy/ini

Figure 7. Time evolution of the transverse emittances in the emittance growth dominated regime $\left(Q_{x}=6.285\right)$ for $I_{o c t}=-20 \mathrm{~A}$. Note that the precision of the emittance measurements is $\sim 10 \%$ [14], and the vertical error bars correspond to one standard deviation of five measurements.

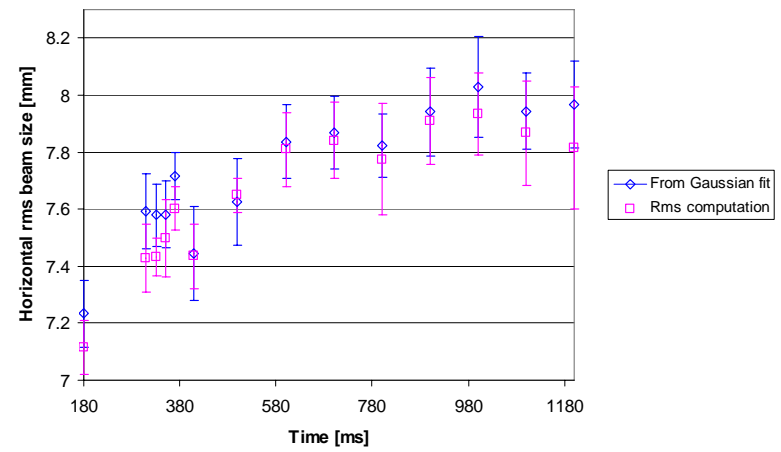

Figure 8. Time evolution of the horizontal rms beam size in the emittance growth dominated regime $\left(Q_{x}=6.285\right)$ for $I_{o c t}=-20 \mathrm{~A}$.

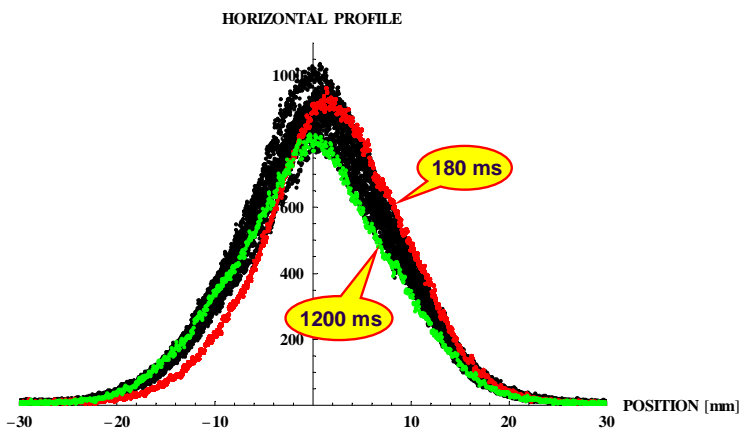

Figure 9. Time evolution of the horizontal profiles in the emittance growth dominated regime.

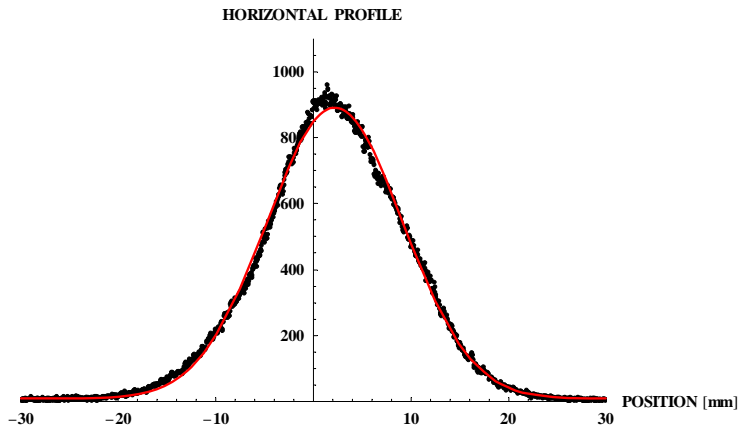

Figure 10. Gaussian fit of the first profile of Fig. 9.

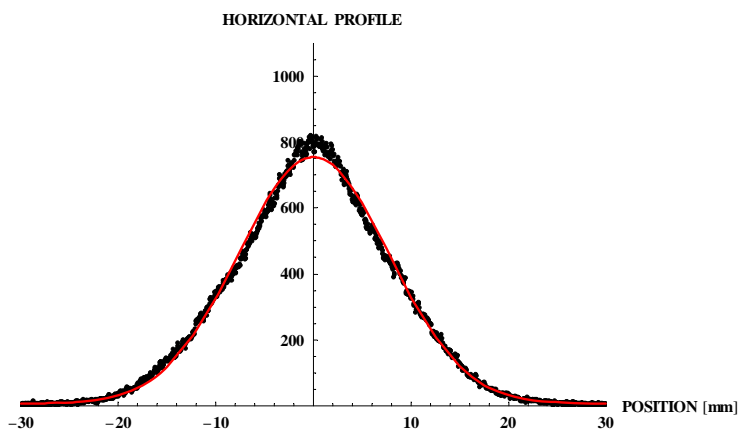

Figure 11. Gaussian fit of the last profile of Fig. 9. 


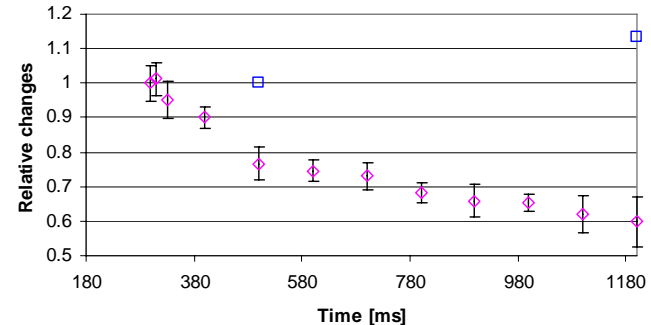

Figure 12. Time evolution of the emittances in the beam loss dominated regime $\left(Q_{x}=6.265\right)$ for $I_{o c t}=-20 \mathrm{~A}$.

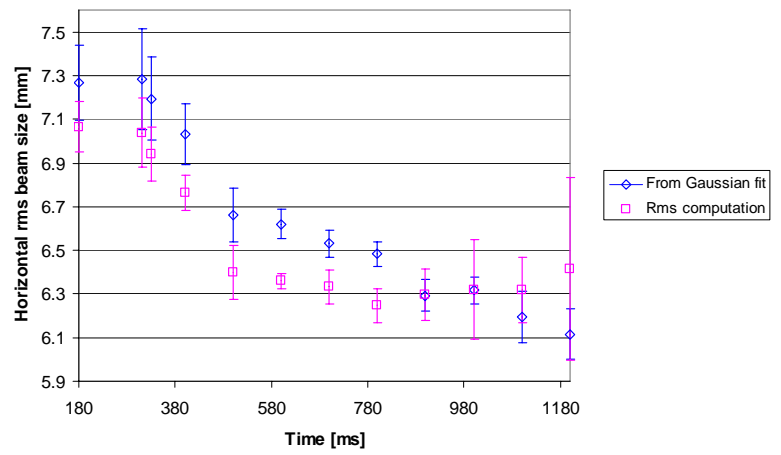

Figure 13. Time evolution of the horizontal rms beam size in the beam loss dominated regime $\left(Q_{X}=6.265\right)$ for $I_{o c t}=-20 \mathrm{~A}$.

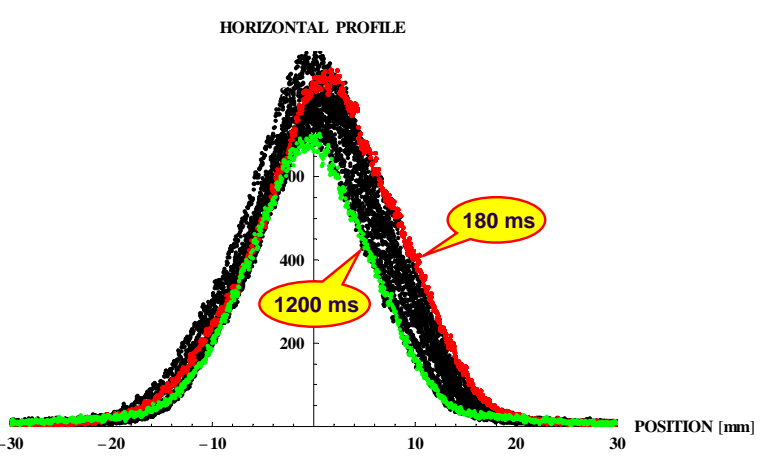

Figure 14. Time evolution of the horizontal profiles in the beam loss dominated regime.

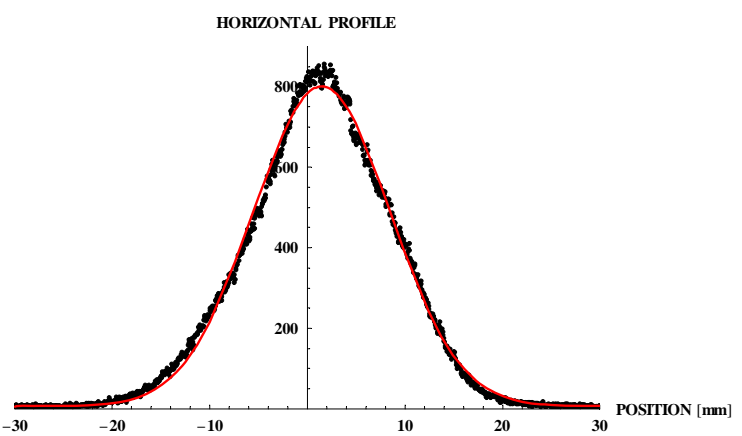

Figure 15. Gaussian fit of the first profile of Fig. 14.

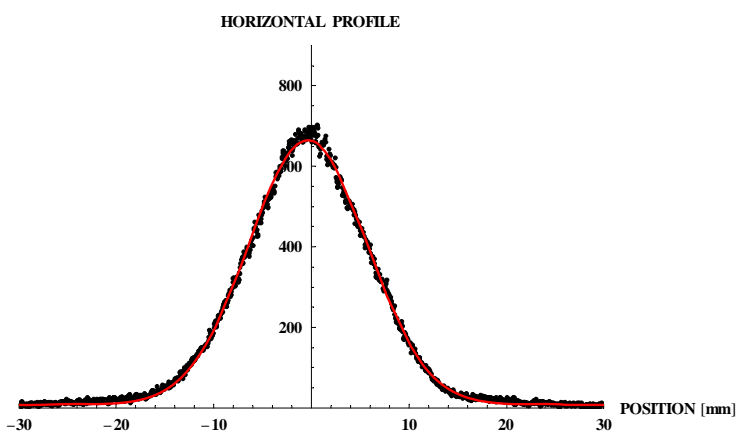

Figure 16. Gaussian fit of the last profile of Fig. 14.

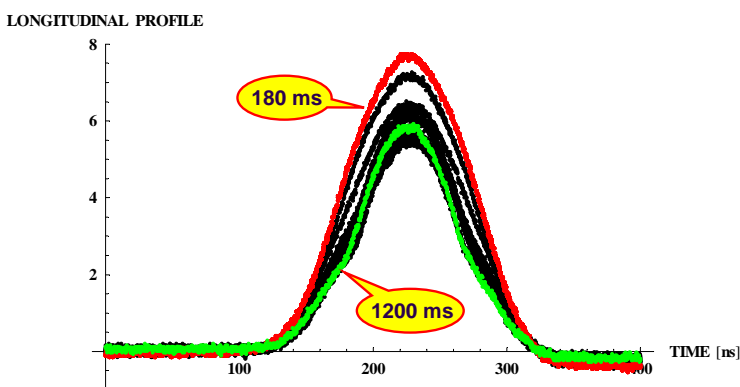

Figure 17. Time evolution of the longitudinal profiles in the beam loss dominated regime. The undershoot on the right is due to the measurement device (see also Figs. 18 and 19).

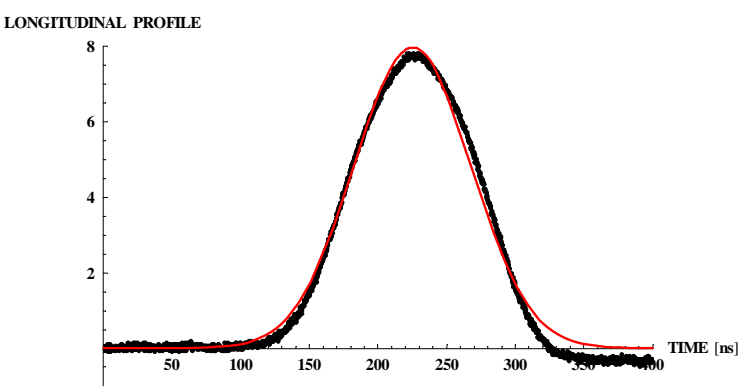

Figure 18. Gaussian fit of the first profile of Fig. 17.

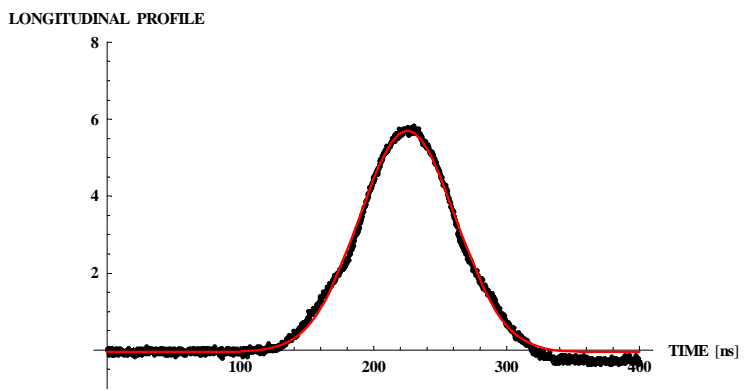

Figure 19. Gaussian fit of the last profile of Fig. 17. 


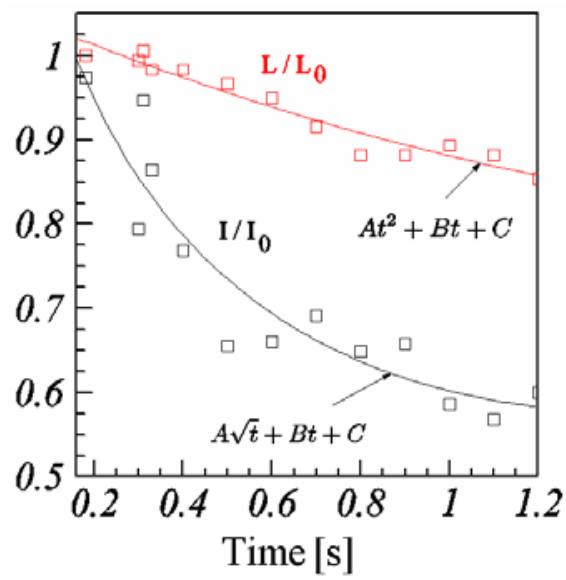

Figure 20. Time evolution of (i) the bunch length in the beam loss dominated regime by doing a Gaussian fit of the longitudinal bunch profiles of Fig. 17, and (ii) the integrated intensity of the longitudinal bunch profiles of Fig. 17.

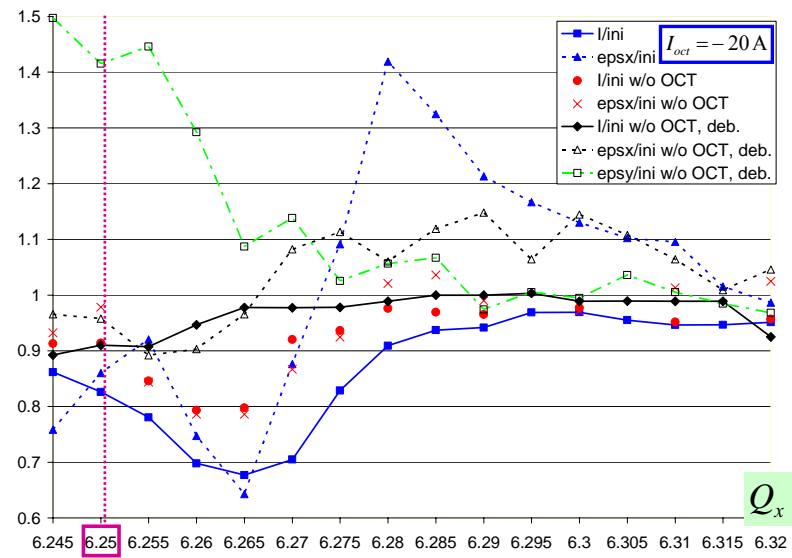

Figure 21. Normalised final (i.e. measured at $1200 \mathrm{~ms}$ ) emittance and beam intensity as function of the working point for the cases (i) with octupole $\mathrm{ON}$ and a current in the octupole of $-20 \mathrm{~A}$, (ii) without (w/o) octupole, and (iii) without (w/o) octupole and with a debunched (deb) beam (i.e. with low space charge).

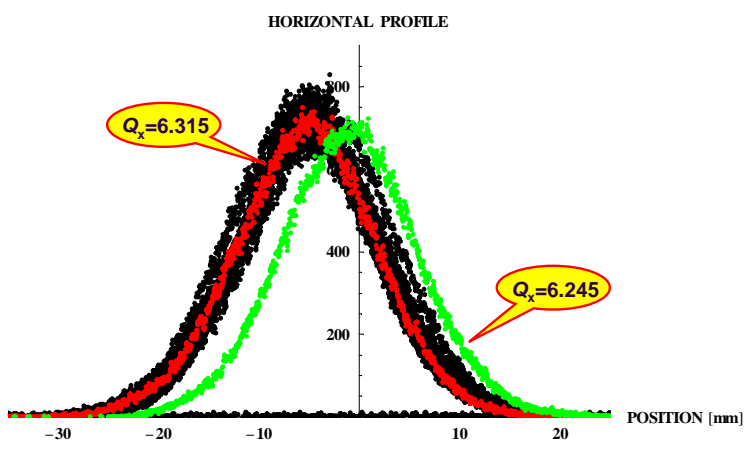

Figure 22. Evolution of the horizontal profiles vs. the horizontal tune for a debunched beam with no octupole.

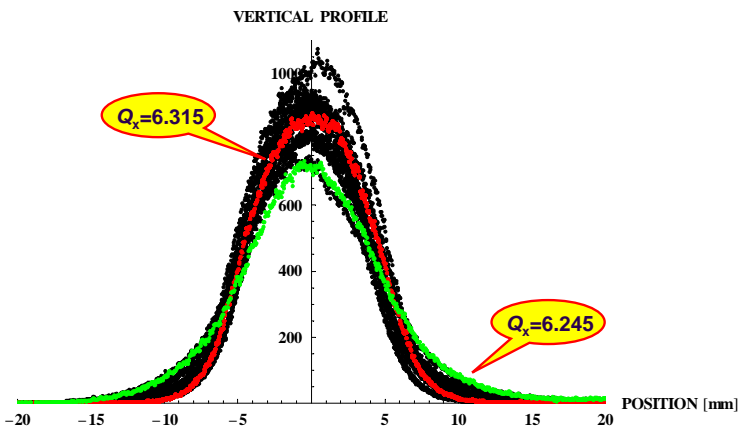

Figure 23. Evolution of the vertical profiles vs. the horizontal tune for a debunched beam with no octupole.

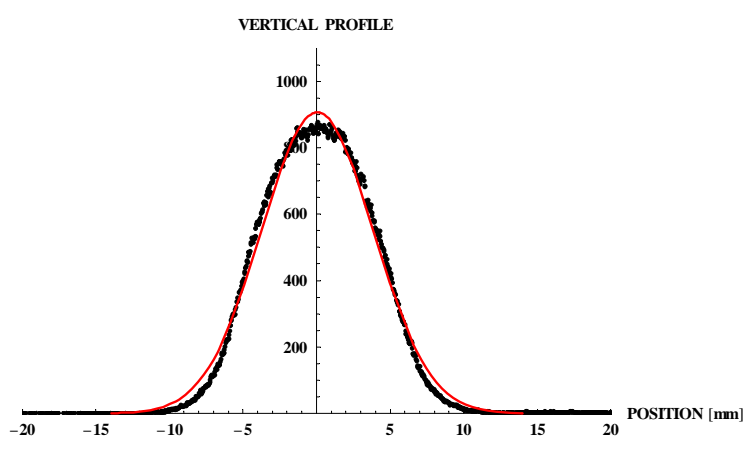

Figure 24. Gaussian fit of the first profile of Fig. 23.

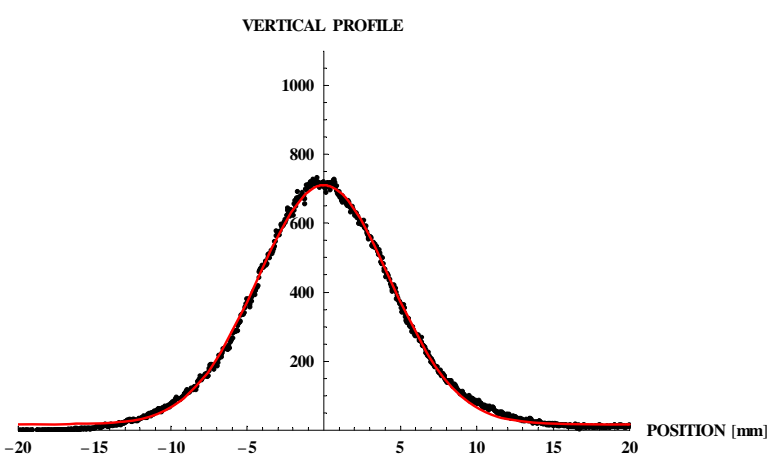

Figure 25. Gaussian fit of the last profile of Fig. 23.

\section{Conclusions and Outlook}

An important contribution to the losses in bunched beams can be explained in terms of particles trapped into lattice and space charge driven islands. This phenomenon involves three mechanisms, namely the space charge tune spread, a nonlinear (octupolar) resonance, and the synchrotron motion. While the octupole is the driving mechanism for the resonance, the detuning effect, as an amplitude limiting mechanism, is not only due to the octupole and other lattice nonlinearities, but mainly due to space charge. Here we are dealing with the transverse amplitude dependence of 
space charge as well as the strong periodic modulation of the transverse tune shift during a synchrotron period, which is the main source of tune modulation in our experiment. Note that chromaticity-dependent tune modulation is more than an order of magnitude lower.

The experimental study in the PS and comparison with simulations has reached a reasonable agreement in the emittance growth regime [2]. The beam loss dominated regime is now better explained than in 2002 (using the nonlinear model of the PS machine instead of the constant focusing approximation). The observed maximum losses $(\sim 30 \%)$ are still larger than predicted $(\sim 8 \%)$. However, with octupole OFF $\sim 20 \%$ of maximum beam losses are already observed, which may explain the difference (see Fig .21). These losses were not observed during the first measurement campaign of 2002, where an octupole current of -20 A was used, instead of $+40 \mathrm{~A}$ in 2003.

Further steps will be needed to get a better quantitative agreement between the observed beam losses and the predictions from simulation, like adding chromaticity [15]. From the experimental point of view, more refined measurements of the nonlinearities for the working points used during these experiments are also necessary.

\section{REFERENCES}

1. M. Giovannozzi et al., "Measurements of Transverse Space-Charge Effects in the CERN Proton Synchrotron", Proc. PAC 2003, Portland, Oregon, USA, 12-16 May 2003.

2. G. Franchetti et al., "Space Charge and Octupole Driven Resonance Trapping Observed at the CERN Proton Synchrotron", Phys. Rev. ST Accel. Beams 6, 124201, December 2003.

3. G. Franchetti et al., "Long Term Simulations of Space Charge and Beam Loss Observed in the CERN Proton Synchrotron", Proc. $33^{\text {rd }}$ ICFA Advanced Beam Dynamics Workshop on High Intensity and High Brightness Hadron Beams, Bensheim, Germany, 18-22 October, 2004.

4. G. Franchetti et al., "Space Charge and Optics Study for High Intensity Machine", $1^{\text {st }}$ CAREHHH-APD workshop on Beam Dynamics in Future Hadron Colliders and Rapidly Cycling HighIntensity Synchrotrons, CERN, Geneva, Switzerland, 8-11 November, 2004.

5. E. Keil, "Non-Linear Space Charge Effects I", CERN/ISR-TH/72-7, 1972.

6. M. Martini, "An Exact Expression for the Momentum Dependence of the Space Charge Tune Shift in a Gaussian Bunch", CERN-PS-93-23-PA, 1993.

7. Y. Wang et al., Phys. Rev. E 49, 5697 (1994).
8. J. Gareyte, A. Hilaire, and F. Schmidt, Proc. of the 1989 IEEE Part. Accel. Conf., Chicago (IEEE, New York, 1989), p. 1376.

9. W. Fischer, M. Giovannozzi, and F. Schmidt, Phys. Rev. E 55, 3507 (1997).

10. T. Satogata et al., Phys. Rev. Lett. 68, 1838 (1992).

11. E. Métral, "Measurement of the PS Linear Coupling using FFT Analysis", CERN/PS/CA/ Note 97-24, 1997.

12. R. Cappi et al., "Optics Studies for the CERN Proton Synchrotron Machine: Linear and Nonlinear Modelling using Beam Based Measurements", Proc. PAC 2003, Portland, Oregon, USA, 12-16 May 2003.

13. G. Arduini et al., "2002 Nonlinear Optics Measurements and Modelling for the CERN SPS", Proc. PAC 2003, Portland, Oregon, USA, 12-16 May 2003.

14. R. Cappi (Editor), Proc. $4^{\text {th }}$ ICFA Beam Dynamics Mini-Workshop on Transverse Emittance Preservation and Measurement, CERN, 5-7 November 1997.

15. G. Franchetti, "Particle Trapping by Nonlinear Resonances and Space Charge", these proceedings. 